Communications in Physics, Vol. 22, No. 2 (2012), pp. 133-140

\title{
THE MAGNETIC CASIMIR EFFECT BETWEEN TWO PARALLEL FERROMAGNETIC PLATES
}

\author{
DO PHUONG LIEN \\ Institute of Engineering Physics (IEP), \\ NGUYEN ANH TUAN* AND NGUYEN TUAN ANH \\ International Training Institute for Materials Science (ITIMS) \\ Hanoi University of Technology (HUT), \\ 1 Dai Co Viet Road, Hai Ba Trung District, Hanoi, Vietnam \\ (*)Corresponding author: tuanna@itims.edu.vn
}

\begin{abstract}
In this work, we study theoretically the long-range magnetic interaction between two parallel ferromagnetic plates, which arises from the magnetic Casimir effect. The behavior of the interaction is discussed for two configurations when the magnetization is perpendicular or parallel to the plates. The dependence of the Casimir force and Casimir energy on different interplate distances $D$ has been investigated. Results of numerical calculations for a cobalt system have been also presented.
\end{abstract}

\section{INTRODUCTION}

The Casimir effect, discovered more than 60 years ago in the seminal paper by Casimir [1], is one of the most direct manifestations of the existence of zeropoint vacuum oscillations. Casimir predicted the existence of an attractive force between two electrically neutral, infinitely large, parallel conducting planes placed in a vacuum. For a long time Casimir's paper remained relatively unknown, but starting from the 1970s it has rapidly received increasing attention.

During the last few years significant progress has been made both in the measurement of the Casimir force and in the development of new calculation methods applicable to nontrivial geometries and taking into account real material properties of the interacting bodies. Accurate measurements have been difficult to be realized because of the difficulty in aligning the plates in precisely parallel position. However, in 2001 a group from the University of Pauda has been able to accurately measure the Casimir force between two parallel plates using micro resonators [2]. This force is a consequence of the fluctuations of the electromagnetic energy in vacuum, and in the presence of surfaces. When changing the boundary condition of the electromagnetic field (e.g by moving a plate with respect to another), the zero-point energy of the system changes and therefore results in an observable force. The boundary condition of the electromagnetic field can, however, also be modified without any mechanic displacement, but rather, by changing the order parameter of a collective ordering phenomenon such as ferromagnetism. When the two plates are ferromagnetic, the magneto-optical Kerr effect influences the boundary condition of the 
electromagnetic field so that the Casimir effect manifests the difference in energy (per unit area);

$$
\Delta E=E_{A F}-E_{F M}
$$

between the configurations in which the two plates have their magnetization antiparallel $(\mathrm{AF})$ or parallel (FM) to each other, i.e. as a magnetic interaction. The dependence of the Casimir force (per unit area) upon the relative orientation of the plates can therefore be derived as:

$$
\Delta F=F_{A F}-F_{F M}=-\frac{d \Delta E}{d D}
$$

where $D$ is the distance between two plates.

\section{GENERAL THEORY}

So far, it exists essentially two kinds of magnetic interaction between magnetic moments or magnetized bodies: the dipole-dipole magneto-static interaction, and the electron-mediated exchange interaction. The former, being a relativistic effect, is weak but long-ranged, and therefore mostly effective at the macroscopic or mesoscopic level. The latter, being due the combined effect of the Coulomb interaction and the Pauli exclusion principle, is much stronger, but typically short ranged, and is the most important interaction at the atomic scale. For two uniformly magnetized ferromagnetic plates held parallel to each other in vacuum, it is shown in paper [3] that the coaction of the Casimir effect and the magneto-optical Kerr effect gives rise to a new long-range magnetic interaction.

The Casimir energy (per unit area) between two plates A and B can be expressed in terms of the reflection coefficients of the plates as [3]

$$
\begin{aligned}
& E=\frac{\hbar}{(2 \pi)^{3}} \int_{0}^{+\infty} d k_{\perp} k_{\perp} \int_{0}^{2 \pi} d \varphi \int_{0}^{k_{\perp} c} d \omega \\
& \times \operatorname{Re} \operatorname{Tr} \log \left[1-R_{A}\left(i \omega, i k_{\perp}, \varphi\right) R_{B}\left(i \omega, i k_{\perp}, \varphi\right) e^{-2 k_{\perp} D}\right]
\end{aligned}
$$

where $\omega$ is the electromagnetic field frequency, $k_{\perp}$ is the component of the wave vector perpendicular to the plates and $\varphi$ is the angle between the incidence plane and $X$-axis (Z-axis being perpendicular to plates). Furthermore, $R_{A(B)}$ are matrices containing the reflection coefficients of the plates:

$$
R_{A(B)}=\left(\begin{array}{ll}
r_{s s}^{A(B)} & r_{s p}^{A(B)} \\
r_{p s}^{A(B)} & r_{p p}^{A(B)}
\end{array}\right)
$$

where the indices $s$ or $p$ correspond to a polarization with the electric field perpendicular or parallel to the incidence plane. We adopt the convention that the $p$ axis remains unchanged upon reflection. All reflection coefficients can be expressed in terms of the diagonal $\varepsilon_{x x}(i \omega)$ and off-diagonal $\varepsilon_{x y}(i \omega)$ components of the dielectric tensor which are evaluated at imaginary frequency of the magnetic field, as following:

$$
\begin{gathered}
r_{s s}\left(i \omega, i k_{\perp}\right)=\frac{k_{\perp} c-\xi}{k_{\perp} c+\xi} \\
r_{p p}\left(i \omega, i k_{\perp}\right)=\frac{\varepsilon_{x x}(i \omega) k_{\perp} c-\xi}{\varepsilon_{x x}(i \omega) k_{\perp} c+\xi}
\end{gathered}
$$




$$
\begin{aligned}
r_{s p}^{\perp}\left(i \omega, i k_{\perp}\right) & =\frac{-\varepsilon_{x y}(i \omega) k_{\perp} c \omega}{\left(k_{\perp} c+\xi\right)\left[\varepsilon_{x x}(i \omega) k_{\perp} c+\xi\right]} \\
r_{s p}^{\|}\left(i \omega, i k_{\perp}\right) & =\frac{-k_{\perp} c \sqrt{\omega^{2}-\left(k_{\perp} c\right)^{2}} \omega \varepsilon_{x y}(i \omega)}{\left(k_{\perp} c+\xi\right)\left[\varepsilon_{x x}(i \omega) k_{\perp} c+\xi\right] \xi} \\
\Delta r_{p p}\left(i \omega, i k_{\perp}\right) & =\frac{2 \sqrt{\omega^{2}-\left(k_{\perp} c\right)^{2}} k_{\perp} c \varepsilon_{x y}(i \omega)}{\left[\varepsilon_{x x}(i \omega) k_{\perp} c+\xi\right]^{2}}
\end{aligned}
$$

where we defined:

$$
\xi=\sqrt{\omega^{2}\left[\varepsilon_{x x}(i \omega)-1\right]+\left(k_{\perp} c\right)^{2}}
$$

\section{Expression for the Casimir energy and force}

Using the reflection coefficients of Eqs. (5)-(9), we will now calculate the Casimir interaction energy and the Casimir force per unit area between the identical plates at $T=0$ when the magnetization is perpendicular to the plates. We call this configuration as polar configuration because this force and energy are related to the polar magneto-optic Kerr effect. They can be expressed as [3]:

$$
\begin{aligned}
& \Delta E^{\perp}=E_{A F}^{\perp}-E_{A M}^{\perp}=-\frac{\hbar}{\pi^{2}} \int_{0}^{+\infty} d k_{\perp} k_{\perp} \int_{0}^{k_{\perp} c} d \omega R e\left[\frac{\left(r_{s p}^{\perp}\right)^{2} e^{-2 k_{\perp} D}}{\left(1-r_{s s}^{2} e^{-2 k_{\perp} D}\right)\left(1-r_{p p}^{2} e^{-2 k_{\perp} D}\right)}\right] \\
& \Delta F^{\perp}=-\frac{d \Delta E^{\perp}}{d D}=-\frac{2 \hbar}{\pi^{2}} \int_{0}^{+\infty} d k_{\perp} k_{\perp}^{2} \int_{0}^{k_{\perp} c} d \omega \times R e\left\{\frac{\left(r_{s p}^{\perp}\right)^{2}\left[1-r_{s s}^{2} r_{p p}^{2} e^{-4 k_{\perp} D}\right] e^{-2 k_{\perp} D}}{\left[\left(1-r_{s s}^{2} e^{-2 k_{\perp} D}\right)\left(1-r_{p p}^{2} e^{-2 k_{\perp} D}\right)\right]^{2}}\right\}
\end{aligned}
$$

For the "in plane configuration" (the magnetization is parallel to the plates), the Casimir energy and force comprise two parts connecting to the longitudinal and transversal Kerr effects which are of opposite signs and therefore tend to cancel each other as following [3]:

$$
\begin{gathered}
\Delta E_{1}^{/ /}=\frac{\hbar}{2 \pi^{2}} \int_{0}^{+\infty} d k_{\perp} k_{\perp} \int_{0}^{k_{\perp} c} d \omega R e\left[\frac{\left(r_{s p}^{/ /}\right)^{2} e^{-2 k_{\perp} D}}{\left(1-r_{s s}^{2} e^{-2 k_{\perp} D}\right)\left(1-r_{p p}^{2} e^{-2 k_{\perp} D}\right)}\right] \\
\Delta E_{2}^{/ /}=-\frac{\hbar}{4 \pi^{2}} \int_{0}^{+\infty} d k_{\perp} k_{\perp} \int_{0}^{k_{\perp} c} d \omega R e\left[\frac{\Delta r_{p p}^{2} e^{-2 k_{\perp} D}}{\left(1-r_{p p}^{2} e^{-2 k_{\perp} D}\right)^{2}}\right] \\
\Delta F_{1}^{/ /}=\frac{\hbar}{\pi^{2}} \int_{0}^{+\infty} d k_{\perp} k_{\perp}^{2} \int_{0}^{k_{\perp} c} d \omega R e\left\{\frac{\left(r_{s p}^{\|}\right)^{2}\left[1-r_{s s}^{2} r_{p p}^{2} e^{-4 k_{\perp} D}\right] e^{-2 k_{\perp} D}}{\left[\left(1-r_{s s}^{2} e^{-2 k_{\perp} D}\right)\left(1-r_{p p}^{2} e^{-2 k_{\perp} D}\right)\right]^{2}}\right\} \\
\Delta F_{2}^{/ /} \approx \frac{-\hbar}{2 \pi^{2}} \int_{0}^{+\infty} d k_{\perp} k_{\perp}^{2} \int_{0}^{k_{\perp} c} d \omega R e\left\{\frac{\Delta r_{p p}^{2}\left[1+r_{p p}^{2} e^{-2 k_{\perp} D}\right] e^{-2 k_{\perp} D}}{\left(1-r_{p p}^{2} e^{-2 k_{\perp} D}\right)^{3}}\right\}
\end{gathered}
$$

It should note that the integral over the angle $\varphi$ in the equation (3) gives $2 \pi$ resulting from the rotational invariance of the two plates around the Z-axis. 


\section{Dielectric tensor}

It is necessary to know the components of dielectric tensor for evaluating the Casimir energy and force. In our paper, these components have been calculated in two models: Drude model and a model called "real model".

\section{Drude model}

In a Drude model, the expressions of the diagonal and off-diagonal parts of the dielectric tensor, evaluated at imaginary frequency of the magnetic field, are written as:

$$
\begin{gathered}
\varepsilon_{x x}(i \omega)=1+\frac{\omega_{P}^{2} \tau}{\omega(1+\omega \tau)} \\
\varepsilon_{x y}(i \omega)=\frac{\omega_{P}^{2} \omega_{c} \tau^{2}}{\omega(1+\omega \tau)^{2}}
\end{gathered}
$$

where the plasma frequency $\omega_{P}$ is defined by $\omega_{P}=\frac{4 \pi n e^{2}}{m *}$; the cyclotron frequency $\omega_{c}$ is given by $\omega_{c}=\frac{e B_{e f f}}{m *}, B_{e f f}$ is the effective magnetic field amplitude; $\tau$ is the relaxation time, $m *$ is the effective mass of the electron.

\section{"Real" model}

The Drude model is incorrect for a real system because it would describe well the off-diagonal element of the dielectric tensor only when ignoring the interband transitions. But these transitions take place at the frequency of the magnetic field of several electronvolts and must be taken in account. For a real system such as cobalt plates, the diagonal elements $\varepsilon_{x x}(\omega)$ and the off-diagonal elements $\varepsilon_{x y}(\omega)$ of the dielectric tensor of the plate can be calculated from the experimental values of the complex index of refraction and the polar Kerr rotation $\theta_{K}$ and the ellipticity $\varepsilon_{K}$. The diagonal elements $\varepsilon_{x x}(i \omega)$ evaluated at imaginary frequency can be determined from the imaginary part of $\varepsilon_{x x}(\omega)$ as in the following equation:

$$
\varepsilon_{x x}(i \omega)=1+\frac{2}{\pi} \int_{0}^{\infty} d \omega^{\prime} \frac{\omega^{\prime} \operatorname{Im} \varepsilon_{x x}\left(\omega^{\prime}\right)}{\omega^{\prime 2}+\omega^{2}}
$$

For the off-diagonal elements $\varepsilon_{x y}(i \omega)$, they can be derived from the real part of $\varepsilon_{x y}(\omega)$ as:

$$
\varepsilon_{x y}(i \omega)=\frac{2}{\omega \pi} \int_{0}^{\infty} d \omega^{\prime} \frac{\omega^{\prime} R e \varepsilon_{x y}\left(\omega^{\prime}\right)}{\omega^{\prime 2}+\omega^{2}}
$$

\section{NUMERICAL CALCULATIONS}

\section{For the general case}

In our numerical calculations, we have chosen the typical values [3]: $\tau=10^{-13} \mathrm{~s}$, $\hbar \omega_{P}=9.85 \mathrm{eV}$ and $\hbar \omega_{c}=5.9 \mathrm{meV}$.

The results of the dependence of the Casimir energy and interaction force between two plates on the inteplate distances $D$ (from $1 \mathrm{~nm}$ to $1 \mu \mathrm{m}$ ) have been presented in Fig. 1(a) and Fig. 1(b) for the in-plane configuration. In Fig. 2(a) and Fig. 2(b), we compare the strength of the energy and the force between the in-plane and polar configurations. 


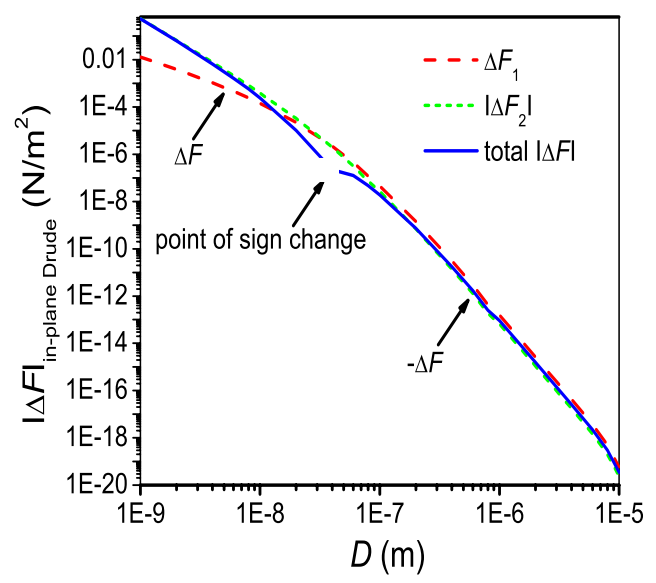

(a)

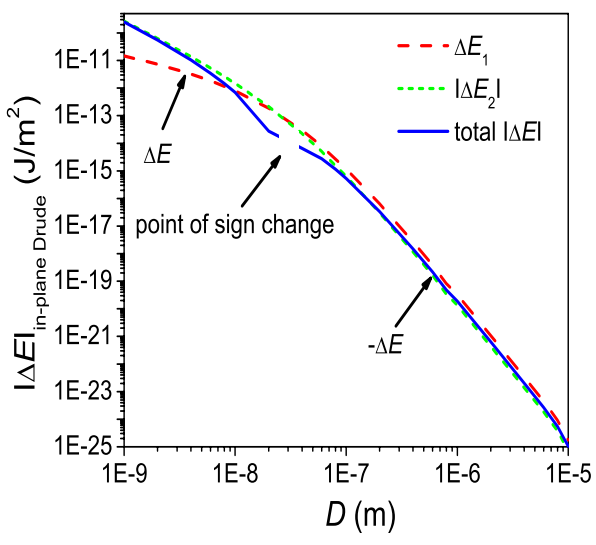

(b)

Fig. 1. Absolute of magnetic Casimir force (a) and energy (b) between the plates calculated in a Drude model for the in-plane configuration.

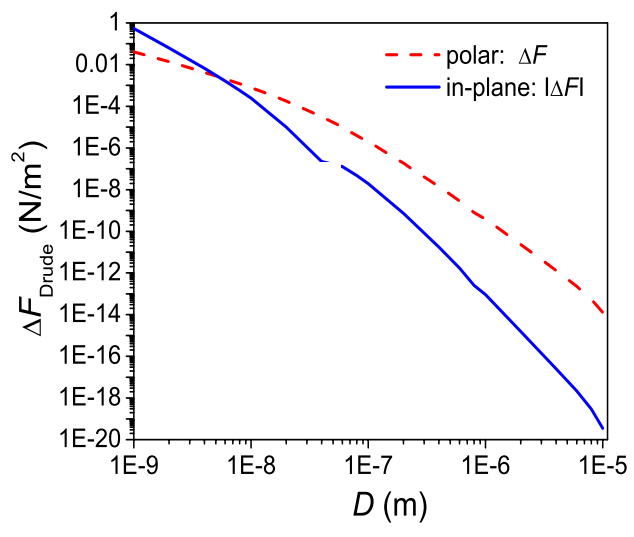

(a)

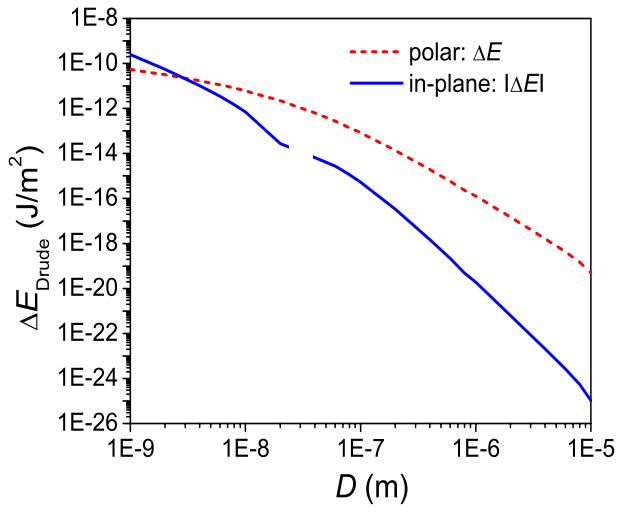

(b)

Fig. 2. Absolute values of magnetic Casimir force (a) and energy (b) per unit area between the plates. The solid curve corresponds to the in-plane configuration while the dashed curve describes the polar configuration.

From two above Figs, we have the following remarks:

- while the values of the energy and force are positive in the polar case, they change their signs when $D$ varies in the case of the in-plane configuration because it comes from the competition of the longitudinal and transversal Kerr effects which are of opposite signs. The point of sign change in the energy (and force) is situated at the distance of $D \approx 40 \mathrm{~nm}$. Therefore, the two plates would 
attract or repulse each other for the in-plane configuration; this fact depends on the interplates distance.

- the amplitude of the force is greater in the in-plane configuration for enough small distances $(D<10 \mathrm{~nm})$

\section{For a system of cobalt plates.}

In the case of cobalt plates, the real and the imaginary parts of the diagonal elements $\varepsilon_{x x}(\omega)$ can be calculated from experimental values of the index of refraction $n$, the extinction coefficient $k$ as:

$$
\operatorname{Re}\left(\varepsilon_{x x}(\omega)\right)=n^{2}-k^{2}, \quad \operatorname{Im}\left(\varepsilon_{x x}(\omega)\right)=2 n k
$$

In the reference [4], the of data of $n$ and $k$ of cobalt is extending from $0.1 \mathrm{meV}$ to $2 \mathrm{keV}$ (corresponding frequencies of the magnetic field are from $10^{12}$ to $10^{19} \mathrm{~s}^{-1}$ ), the imaginary part of diagonal elements $\varepsilon_{x x}(i \omega)$ at imaginary frequency calculated by Eq. (18) in this range is shown in Fig. 3. We have found that their decay law is of $1 /\left[\omega^{0.2}(1+\omega \tau)\right]$.

The experimental data of the off-diagonal elements $\varepsilon_{x y}(\omega)$ is very limited, these elements could be derived from experimental values of the polar Kerr rotation $\theta_{K}$ and the ellipticity $\varepsilon_{K}$ coupled with the diagonal elements $\varepsilon_{x x}(\omega)$ as in the equation below [4]:

$$
\theta_{K}(\omega)+i \varepsilon_{K}(\omega)=\frac{-\varepsilon_{x y}(\omega)}{\varepsilon_{x x}^{1 / 2}(\omega)\left[1-\varepsilon_{x x}(\omega)\right]}
$$

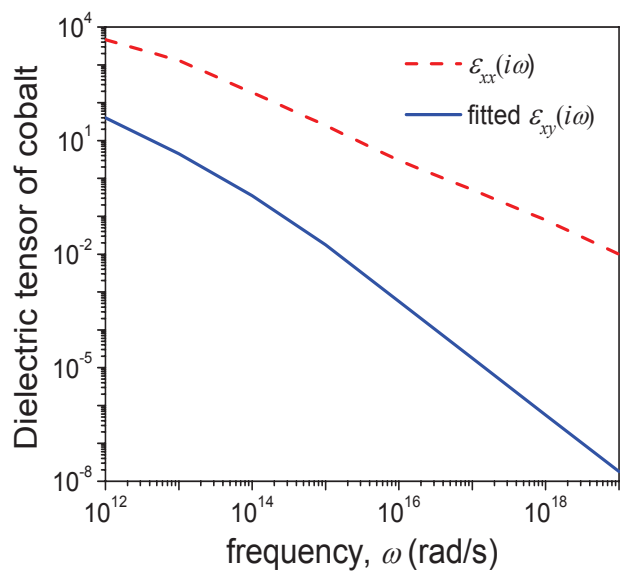

Fig. 3. The elements of the dielectric tensor of cobalt as a function of imaginary frequency

The experimental data for cobalt is available only for the range from $1.5 \mathrm{eV}$ to 4.5 $\mathrm{eV}$. The imaginary part of off-diagonal elements $\varepsilon_{x y}(i \omega)$ at imaginary frequency is then calculated by Eq. (11) in this range. For others frequencies, we made a fit of the curve by another one which decays as $1 / \omega^{2}$, and it is also shown in Fig. 3.

We can remark that while the diagonal elements, $\varepsilon_{x x}(i \omega)$, and off-diagonal elements, $\varepsilon_{x y}(i \omega)$, of the dielectric tensor are inversely proportional to the quadratic polynomial and 
cubic polynomial of frequency $\omega$ in the Drude model, they are proportional respectively to $1 / \omega^{3} / 2$, and $1 / \omega^{2}$, for a cobalt system. This difference in powerlaw can be explained by the fact that the interband transitions are negligible in the Drude model but they exist in a real system such as cobalt.

The magnetic Casimir force and energy can be now calculated by numerical integration of Eqs. (10) and (11). We are interested in plate separations between $1 \mathrm{~nm}$ and 10 $\mu \mathrm{m}$ which correspond to energies of the magnetic field in the range $0.01 \mathrm{eV}-100 \mathrm{eV}$, so we have performed the integration between $10^{-4} \mathrm{eV}$ and $10^{3} \mathrm{eV}$ (corresponding frequencies of the magnetical field are from $10^{12}$ to $10^{19} \mathrm{~s}^{-1}$ ). We have used $\tau=10^{-13} \mathrm{~s}$ as in the case of the Drude model. The figures 4 show the force and energy per unit area for the polar and in-plane configurations. A change of sign of the interaction for the in-plane configuration is also visible from the figure by the discontinuity at $D \approx 400 \mathrm{~nm}$. We can also see that the effect is always larger for the polar configuration.

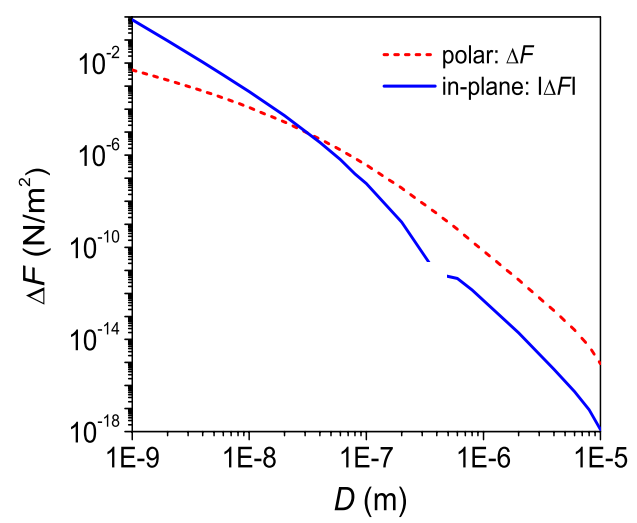

(a)

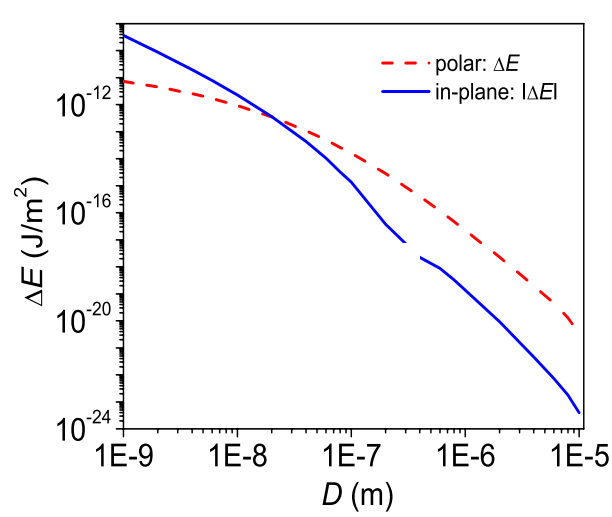

(b)

Fig. 4. Absolute values of magnetic Casimir force (a) and energy (b) per unit area between two cobalt plates. The solid curve corresponds to the in-plane configuration, while the dashed curve describes the polar configuration.

\section{CONCLUSION}

The paper has presented a theoretical approach for calculating the Casimir energy and the interaction between two magnetic plates. The Drude model has been used for the general case. For this case, a number of numerical simulations have been realized, the dependence of the force and energy on the interplate distance has been calculated and the change of sign of the interaction when this distance varies has been observed. We have also carried out numerical calculations for a real system with cobalt plates using the experimental data for the dielectric tensor of cobalt. We found that the power law of the dielectric tensor of cobalt was different from one of the Drude model because interband transitions which haven't been treated correctly in the Drude model became important in 
a Co system. However, the experimental data on the off-diagonal elements of dielectric tensor for ferromagnetic materials are still limited, which certainly affect the results.

We would like to study more systems with this theoretical approach and even again for cobalt but with a more accurate model for the dielectric tensor.

\section{ACKNOWLEDGMENTS}

This work was supported by the National Foundation for Science and Technology Development (NAFOSTED) of Vietnam under Project Code No. 103.02.50.09.

\section{REFERENCES}

[1] H. B. G. Casimir, Proc. K. Ned. Akad. Wet. 51 (1948) 793.

[2] G. Bressi, G. Carugno, R. Onofrio, G. Russo, Phys. Rev. Lett. 88 (2002) 041804.

[3] G. Metalidis and P. Bruno, Phys. Rev. A66 (2002) 062102.

[4] Handbook of Optical Constants of Solids II, edited by Edward D. Palik, Elsevier Science, 1998.

Received 15 October 2010. 